\title{
Quantitative approach to small-scale nonequilibrium systems
}

\author{
Dreyer, Jakob K; Berg-Sørensen, Kirstine; Oddershede, Lene B
}

Published in:

Physical Review E

Link to article, DOI:

10.1103/PhysRevE.73.051110

Publication date:

2006

Document Version

Publisher's PDF, also known as Version of record

Link back to DTU Orbit

Citation (APA):

Dreyer, J. K., Berg-Sørensen, K., \& Oddershede, L. B. (2006). Quantitative approach to small-scale nonequilibrium systems. Physical Review E, 73(5), 051110. https://doi.org/10.1103/PhysRevE.73.051110

\section{General rights}

Copyright and moral rights for the publications made accessible in the public portal are retained by the authors and/or other copyright owners and it is a condition of accessing publications that users recognise and abide by the legal requirements associated with these rights.

- Users may download and print one copy of any publication from the public portal for the purpose of private study or research.

- You may not further distribute the material or use it for any profit-making activity or commercial gain

- You may freely distribute the URL identifying the publication in the public portal 


\title{
Quantitative approach to small-scale nonequilibrium systems
}

\author{
Jakob Kisbye Dreyer, ${ }^{1, *}$ Kirstine Berg-Sørensen, ${ }^{2,1, \dagger}$ and Lene Oddershede ${ }^{1, \ddagger}$ \\ ${ }^{1}$ Niels Bohr Institute, Blegdamsvej 17, DK-2100 Copenhagen, Denmark \\ ${ }^{2}$ Department of Physics, Technical University of Denmark, DK-2800 Lyngby, Denmark \\ (Received 14 June 2005; revised manuscript received 17 March 2006; published 24 May 2006)
}

\begin{abstract}
In a nanoscale system out of thermodynamic equilibrium, it is important to account for thermal fluctuations. Typically, the thermal noise contributes fluctuations, e.g., of distances that are substantial in comparison to the size of the system and typical distances measured. If the thermal fluctuations are ignored, misinterpretation of measured quantities such as interaction forces, potentials, and constants may result. Here, we consider a particle moving in a time-dependent landscape, as, e.g., in an optical tweezers or atomic force nanoscopic measurement. Based on the Kramers equation [H. A. Kramers, Physica 7, 284 (1940)], we propose an approximate but quantitative way of dealing with such an out-of-equilibrium system. The limits of this approximate description of the escape process are determined through optical tweezers experiments and comparison to simulations. Also, this serves as a recipe for how to use the proposed method to obtain knowledge about the underlying energy landscape from a set of experimental measurements. Finally, we perform estimates of the error made if thermal fluctuations are ignored.
\end{abstract}

DOI: 10.1103/PhysRevE.73.051110

PACS number(s): 05.40.-a, 34.20.-b, 82.20.Uv, 82.70.Dd

\section{INTRODUCTION}

Most of our knowledge about the physical world originates from systems in thermodynamic equilibrium. However, with the increased interest in nanoscale systems and the advance of nanoscopic techniques such as atomic force microscopy or optical tweezers, it becomes increasingly important to know how to deal quantitatively with systems that are not in thermal equilibrium and for which thermal fluctuations cannot be ignored. The equations of Jarzynski [1] and Crooks [2] relate the nonequilibrium state of a system to equilibrium thermodynamics and have been tested, e.g., through studies of biological processes [3,4]. Here, we demonstrate an alternative method based on the Kramers equation [5].

We address the situation where a micro- or nanoscale system is caught in a local energy minimum with a timeevolving energy barrier and we study the transition from the metastable state to the equilibrium configuration. A fundamental problem in this type of experiment is that the system is not in thermal equilibrium and hence it is not well described by conventional thermodynamics. Experimentally, we consider a particle trapped by optical tweezers while approaching an attractive wall until the particle escapes the optical trap and jumps to the surface. This scenario constitutes a time-evolving bistable energy landscape with the optical tweezers potential as a metastable state; the closer the optical trapping position is to the wall, the lower the energy barrier separating the two states. Based on Kramers theory we investigate this situation, and show how to obtain infor-

\footnotetext{
*Present address: Device Chemistry and Biology, Novo Nordisk, DK 3400 Hillerød, Denmark. Electronic address: jkid@novonordisk.com

†Electronic address: kirstine.berg-sorensen@fysik.dtu.dk

*Corresponding author. Electronic address: oddershede@nbi.dk
}

mation about the underlying interaction forces. The validity of this approach is tested by simulations and by comparison to experiments. Most importantly, our work provides a test bed for a quantitative treatment of small-scale systems out of equilibrium, systems for which thermal fluctuations play a crucial role. This work is particularly important for singlemolecule biophysics, cell biology, and the nanosciences in general.

\section{EXPERIMENTS}

The experimental test system was a colloid held by optical tweezers and gradually approaching a glass surface. Due to the attractive van der Waals force, the colloid and glass surface experienced an attractive interaction. The colloid was of polystyrene with diameter $1.07 \mu \mathrm{m}$ suspended in $10 \mathrm{mM}$ $\mathrm{NaCl}$ solution. Eventually, the colloid would escape the trap and jump to the surface. A typical jump event is depicted in Fig. 1(a). The jump is observed at $t \simeq 9 \mathrm{~s}$. The equilibrium position of the trapping potential is denoted $z_{0}$ and $z$ is the distance from the bead to the surface. The length of the jump ( $157 \mathrm{~nm}$ for the data shown) is defined as the value of $z_{0}$ at the time of the jump. Before the jump, the colloid underwent thermal fluctuations within the trapping potential. After the jump, the thermal motion of the bead was greatly reduced. We interpret this as the bead being irreversibly bound to the glass surface. The approach speed of the optical trap is constant and denoted $v$. The trap constitutes a three-dimensional harmonic potential and lateral fluctuations of the bead do not change its escape probability in the axial direction. The optical tweezers setup is described in Refs. [6,7] and the colloid-surface measurements are described in Ref. [8]. The force constant in the escape direction $\kappa=0.0092 \mathrm{pN} / \mathrm{nm}$ is found using the methods described in Refs. [8,9].

In order to investigate how the lengths of the jumps depend on approach velocity, experiments were performed at different approach velocities: $v=10,20,40,80 \mathrm{~nm} / \mathrm{s}$. For 

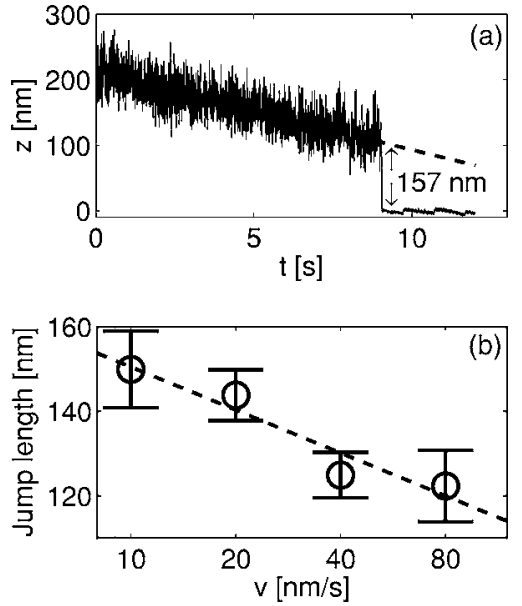

FIG. 1. (a) An optically trapped colloid approaching a surface. The measured height of the colloid over the surface is shown as a function of time. A $157 \mathrm{~nm}$ jump from the equilibrium position of the trap to the surface is observed at $t \simeq 9 \mathrm{~s}$. (b) Average jump length as function of approach velocity.

each velocity, 10-20 jumps were recorded, using a new bead for each experiment. The average jump length for each approach velocity is shown in Fig. 1(b). Here, the typical jump length is found to depend on the approach velocity. This illustrates the probabilistic nature of the escape process.

\section{THEORETICAL MODEL}

To gain detailed understanding of the escape process, the experimental observations are compared to computer simulations and approximative analytical considerations. We assumed the total energy landscape experienced by the particle to be given as

$$
V(z)=\frac{\kappa}{2}\left(z-z_{0}\right)^{2}-\frac{A R}{6 z} .
$$

The first term is the harmonic potential of the optical trap; $\kappa$ denotes the stiffness of the optical trap. The second term is the interaction potential between the surface and the particle as given by the Derjaguin approximation [10]. The Hamaker constant $A$ is on the order of $10^{-20} \mathrm{~J}$ and $R$ is the radius of the bead. For $z_{0}$ larger than a critical value denoted $z_{c}$, both a local minimum, denoted $z_{1}$, and a local maximum, denoted $z_{2}$, exist. The value of $z_{c}$ is calculated below.

The total energy landscape experienced by the trapped particle is shown by a solid line in Fig. 2 in a case where $z_{0}>z_{c}$. The apparatus and interaction contributions to the energy are shown as dotted and dashed lines, respectively. The escape process of the particle is a transition from the local minimum $z_{1}$ over the energy barrier $z_{2}$ to the ground state at $z=0$.

In order to determine $z_{c}$, we rewrite Eq. (1) in terms of the force exerted on the particle,

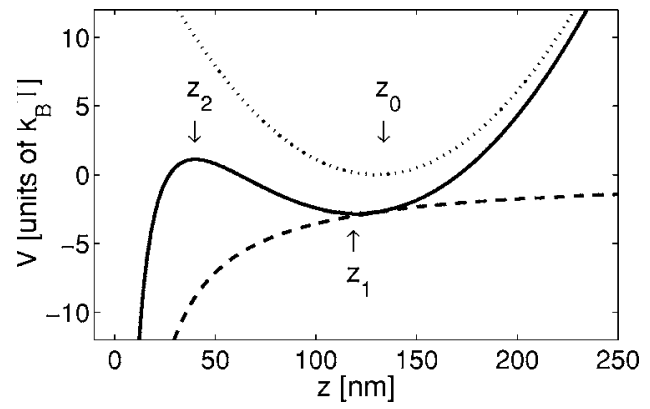

FIG. 2. The interaction potentials used in Eq. (1), with $\kappa=0.01 \mathrm{pN} / \mathrm{nm}$, and $A R / 6=1440 \mathrm{pN} \mathrm{nm}^{2}$. Dotted line: Harmonic potential of the measurement apparatus. Dashed line: van der Waals surface interaction energy between a colloid and a planar surface. Solid line: Total interaction energy from Eq. (1), sum of dotted and dashed lines.

$$
F(z)=-\kappa\left(z-z_{0}\right)-\frac{A R}{6 z^{2}}
$$

as a function of the position $z$. At the bottom of the local energy minimum $z_{1}$ and at the peak of the energy barrier $z_{2}$ the total force vanishes. Solving for $F(z)=0$ is equivalent to solving the third-order polynomial equation

$$
\mathcal{P}(z)=z^{3}-z_{0} z^{2}+\beta=0,
$$

with $\beta$ shorthand for $\frac{A R}{6 \kappa}$. For high values of $z_{0}$ this equation has three real roots, two of which correspond to $z_{1}$ and $z_{2}$ in Fig. 2. The third solution $z_{3}$ is negative and has no physical significance. However, for $z_{0}$ below $z_{c}$, the solutions to Eq. (3) are complex. Instead of solving Eq. (3) for arbitrary values of $z_{0}$ and $\beta$, we seek the special case when $z_{0}=z_{c}$. This is the case where the energy barrier has just vanished and the two roots $z_{1}$ and $z_{2}$ have merged together into a double root denoted $\zeta$. Formally, we may rewrite $\mathcal{P}(z)$ in the form

$$
z^{3}-z_{0} z^{2}+\beta=(z-\zeta)^{2}\left(z-z_{3}\right)+\mathcal{R}(z) .
$$

The remainder is given as

$$
\mathcal{R}(z)=\frac{A z+B}{(z-\zeta)^{2}}
$$

and with $A=2 \zeta\left(2 \zeta-z_{0}\right)-\zeta^{2}$ and $B=-\left(2 \zeta-z_{0}\right) \zeta^{2}+\beta$. In the case when $z_{0}=z_{c}$, the polynomial of Eq. (3) has a double root located in $\zeta$ and hence $\mathcal{R}(z)$ must be 0 for all $z$. This, in turn, leads to $A=B=0$. From these two equations, the unknowns $\zeta$ and $z_{c}$ can be deduced. The result is

$$
\begin{gathered}
\zeta=\frac{2}{3} z_{c}, \\
z_{c}=\left(\frac{9 A R}{8 \kappa}\right)^{1 / 3} .
\end{gathered}
$$

Using the experimentally determined values of $A$ and $\kappa$, we get $z_{c} \sim 100 \mathrm{~nm}$. 


\section{SIMULATIONS}

The simulations of the approach process were based on the Langevin equation. In each time step $\delta t=1 \mathrm{~ms}$, the position $z_{i}$ of the particle was updated according to

$$
z_{i+1}=z_{i}+\left\{-\frac{A R}{6} z_{i}^{-2}-\kappa\left[z_{i}-\left(Z_{0}-v t\right)\right]+\mathcal{F}_{i}\right\} \frac{\delta t}{\gamma} .
$$

Here, we have substituted $z_{0}(t)=Z_{0}-v t$ for the position of the apparatus at time $t$ where $Z_{0}$ is the initial position, which was set to $450 \mathrm{~nm}$. The random driving force $\mathcal{F}_{i}$ is drawn from a Gaussian distribution with $\left\langle\mathcal{F}_{i}^{2}\right\rangle$ in accordance with the fluctuation-dissipation theorem. The viscous drag coefficient $\gamma$ is found from Stokes law with corrections depending on the distance from the particle to the surface [11].

The aim is to extract information about the underlying interactions from the jump data and the escape probability must be related to the features of the energy landscape. A simple quantitative description of this scenario is given by Kramers formula [5] where the escape rate $r$ is given by

$$
r=f_{0} \exp (-\Delta V) \text {. }
$$

Here, $\Delta V=V\left(z_{2}\right)-V\left(z_{1}\right)$ is the height of the energy barrier in units of $k_{B} T$ where $k_{B}$ is Boltzmann's constant and $T$ is the absolute temperature. The attempt frequency $f_{0}$ equals $\frac{\sqrt{k_{1} k_{2}}}{2 \pi \gamma}$ where $k_{1}$ and $k_{2}$ are the curvatures of $V$ evaluated in $z_{1}$ and $z_{2}$.

We now consider a situation where $z_{0}$ decreases linearly, corresponding to the experiment. Initially, the probe is trapped in the local energy minimum located far from the surface where $r \rightarrow 0$. In the experiment the minimum of the trapping potential continuously approaches the surface, i.e., $z_{0}$ decreases. Therefore, $\Delta V$ is decreasing as function of time and the escape probability gradually increases until, finally, the probe escapes from the local minimum and jumps to the surface. To find the distribution of jumps, Kramers equation is integrated numerically. As an alternative to the numerical integration, we also consider an approximation of the energy landscape; this is described below.

\section{SOLVING KRAMERS EQUATION}

The Kramers equation cannot be integrated analytically in the time-evolving energy landscape. In order to find an approximate analytical solution, we linearize the activation energy around the most likely escape distance $z^{*}$,

$$
\Delta V\left(z_{0}\right) \approx \alpha\left(z_{0}-z^{*}\right)+\Delta V\left(z^{*}\right) \equiv \alpha\left(z_{0}-z^{*}\right)+\Delta V^{*} .
$$

The constant $\alpha$ describes how many units of $k_{B} T$ the activation energy $\Delta V$ decreases when $z_{0}$ decreases by one unit of length. Further, $f_{0}$ is assumed to be constant and we have introduced the shorthand notation $\Delta V^{*}$ for $\Delta V\left(z^{*}\right)$. With this approximation, the time-evolving Kramers problem is solved as follows.

The dynamical change in the energy landscape is given by $z_{0}(t)=-v t$ where $v>0$ is the constant approach speed. The probability of not escaping the local minimum at time $t$ is denoted $P(t)$. It satisfies

$$
\frac{d P}{d t}=-r(t) P(t)
$$

The escape rate $r$ is given by the Kramers equation Eq. (9). The evolving energy landscape continuously changes the escape probability $r$. Equation (11) can be rewritten in terms of the apparatus position $z_{0}$ :

$$
H\left(z_{0}\right) \equiv \frac{d P}{d z_{0}}=v^{-1} r\left(z_{0}\right) P\left(z_{0}\right) .
$$

Thus, $P\left(z_{0}\right)$ now denotes the probability of not having escaped the local minimum when the optical trap has reached position $z_{0} . H\left(z_{0}\right)$ is then the probability distribution of jump lengths.

The most general solution to Eq. (12) is the integral

$$
P\left(z_{0}\right)=\exp \left[v^{-1} \int_{+\infty}^{z_{0}} r(z) d z\right] \text {. }
$$

Since the general $z$ dependence of $r$ is complicated, this integral cannot be solved without simplifying assumptions. We use the linearization assumption Eq. (10) as ansatz and the Kramers equation Eq. (9) becomes

$$
r\left(z_{0}\right)=f_{0} \exp \left[-\alpha\left(z_{0}-z^{*}\right)-\Delta V^{*}\right] .
$$

This relation expresses the escape probability $r$ as function of trap position $z_{0}$ and of the parameters of the energy landscape. We note that Eq. (14) does not involve the dynamical change brought in by $z_{0}=-v t$. However, $z^{*}$ and $\alpha$ refer to observables from a dynamic measurement and depend on $v$, as will be shown below. Using the linearized expression in Eq. (14), the integral in Eq. (13) can be solved and $P\left(z_{0}\right)$ becomes

$$
P\left(z_{0}\right)=\exp \left[-\frac{f_{0}}{v \alpha} e^{-\alpha\left(z_{0}-z^{*}\right)-\Delta V^{*}}\right] .
$$

Differentiating with respect to $z_{0}$ gives the distribution of jump lengths,

$$
H\left(z_{0}\right)=\frac{f_{0}}{v} \exp \left[-\frac{f_{0}}{v \alpha} e^{-\alpha\left(z_{0}-z^{*}\right)-\Delta V^{*}}-\alpha\left(z_{0}-z^{*}\right)-\Delta V^{*}\right] .
$$

This expression may be simplified further: Since $z^{*}$ is defined as the most likely jump length, it must satisfy

$$
\left.\frac{\partial H\left(z_{0}\right)}{\partial z_{0}}\right|_{z_{0}=z^{*}}=0 \text {. }
$$

From Eq. (16), we find

$$
\frac{\partial H}{\partial z_{0}}=H\left(z_{0}\right)\left[\frac{f_{0}}{v} e^{-\alpha\left(z_{0}-z^{*}\right)-\Delta V^{*}}-\alpha\right] .
$$

Equation (17) can only be satisfied if

$$
e^{-\Delta V^{*}}=\frac{v \alpha}{f_{0}}
$$

or equivalently 


$$
\Delta V^{*}=\ln \frac{f_{0}}{v \alpha},
$$

thus the typical energy barrier at the time of the escape is seen to depend directly on the approach velocity $v$.

$\Delta V\left(z_{0}\right)$ varies as $\alpha z_{0}$; therefore Eq. (20) implies that

$$
z^{*} \propto-\frac{1}{\alpha} \ln v
$$

when the variation of $z^{*}$ is small.

Substitution of Eq. (20) into Eq. (16) yields the probability distribution of the jump lengths as function of trap positions, $H\left(z_{0}\right)$, expressed by the parameters $\alpha$ and $z^{*}$ :

$$
H\left(z_{0}\right) \approx \alpha \exp \left[-\alpha\left(z_{0}-z^{*}\right)-e^{-\alpha\left(z_{0}-z^{*}\right)}\right] .
$$

The distribution in Eq. (22) is known as the Fisher-TippetGumbel distribution and is also connected with the distribution of the extreme values of the particle's energy in a certain time interval. For a particle trapped in a harmonic potential, the autocorrelation time is given by $\tau=\frac{\gamma}{\kappa}$. From extreme value statistics it is known that the most likely value of the largest energy fluctuation of the particle in a time series of length $\mathcal{T} \gg \tau$ is given by [12]

$$
E_{\max }=\ln (\mathcal{T} \tau) .
$$

Here, we assume that data points recorded at times larger than a time interval $\tau$ apart are statistically independent. Equation (23) may be compared to Eq. (20). If we identify the attempt frequency with the inverse of $\tau, f_{0} \sim \tau^{-1}$, we find that the typical height of the energy fluctuation that carries the particle over the energy barrier is equal to the typical extreme value of the energy in a time interval of length $\mathcal{T}^{*}=(\alpha v)^{-1}$. This characteristic time is the time it takes $\Delta V$ to decrease by $k_{B} T$.

Alternatively, $H\left(z_{0}\right)$ can be mapped directly into the distribution of extreme energy fluctuations in this time interval using the linear mapping of Eq. (10).

\section{COMPARING EXPERIMENT, SIMULATION, AND MODEL}

A comparison between the Langevin simulation Eq. (8), Kramers equation Eq. (9), and the approximation Eq. (22), is shown in Fig. 3. The circles represent a histogram of jump lengths obtained from Langevin simulations at $v=20 \mathrm{~nm} / \mathrm{s}$. The solid line indicates the distribution obtained by numerical integration of Kramers equation. This result is obtained using no free parameters and is overall in good agreement. The dashed line shows a fit of Eq. (22) with $z^{*}=121 \mathrm{~nm}$ and $\alpha \simeq 0.18 \mathrm{~nm}^{-1}$. The numerically integrated result peaks at $z^{*}=123 \mathrm{~nm}$. Similarly, Eq. (22) was used to find $z^{*}$ from other simulated data sets for a range of approach velocities from 1 to $160 \mathrm{~nm} / \mathrm{s}$. The inset of Fig. 3 shows a comparison between $z^{*}$ obtained from a numerical integration of Kramers equation and $z^{*}$ obtained from the Langevin simulation; at low velocities the agreement is excellent. However, at increasing $v$, Kramers equation tends to overestimate the jump length: At $v=160 \mathrm{~nm} / \mathrm{s}$ the deviation is $\sim 10 \mathrm{~nm}$ and is simi-

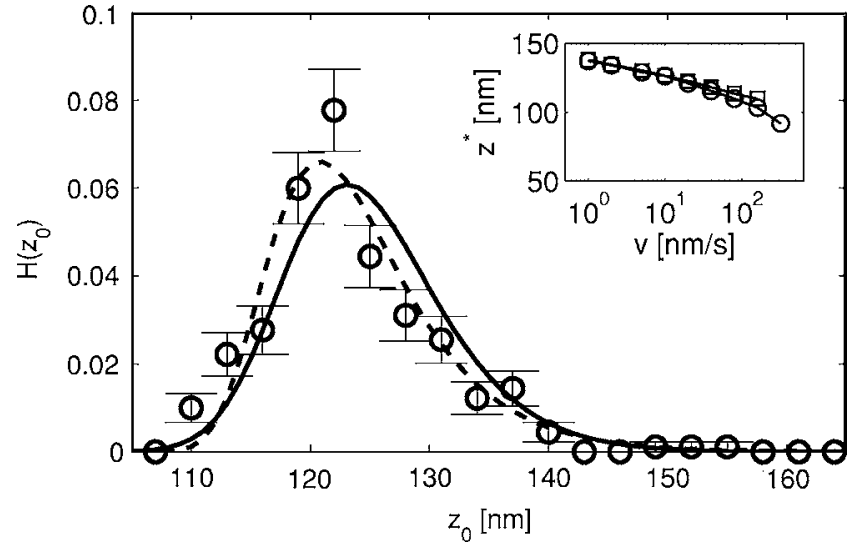

FIG. 3. Predictions of the jump distances using $\kappa=0.01 \mathrm{pN} / \mathrm{nm}$, and $A R / 6=1440 \mathrm{pN} \mathrm{nm}^{2}$ (as in Fig. 2). Circles show a histogram of jump lengths from a Langevin simulation with $v=20 \mathrm{~nm} / \mathrm{s}$. Solid line: Numerical integration of Kramers equation Eq. (9). Dashed line: Fit of Eq. (22) to the simulation data. Inset: Most likely jump length $z^{*}$ as function of approach velocity. Squares indicate numerical integration of Kramers equation and circles Langevin simulation.

lar to the experimental uncertainty. This reflects that Kramers equation is derived under the assumption of quasistationarity. Thus, Eq. (9) only holds when $\Delta V \gg 1$, that is, when the approach velocity is sufficiently low.

A histogram of jumps lengths obtained experimentally is shown in Fig. 4. The solid line shows a fit using Eq. (22) with parameters $z^{*}=132 \mathrm{~nm}$ and $\alpha=0.09 \mathrm{~nm}^{-1}$. The dashed line represents a Gaussian fit with mean $137 \mathrm{~nm}$ and standard deviation $14 \mathrm{~nm}$. Calculations of $\chi^{2}=1.0$ for the fit of Eq. (22) and for a Gaussian fit $\left(\chi^{2}=1.4\right)$ show that Eq. (22) is slightly better. This, in addition to the fact that the variation of $z^{*}$ with the logarithm of $v$ from the experiment (circles in the inset of Fig. 4) appears linear, as predicted by Eq. (21), constitutes a comparison between the experiments and model. The agreement is definitely satisfactory for the ve-

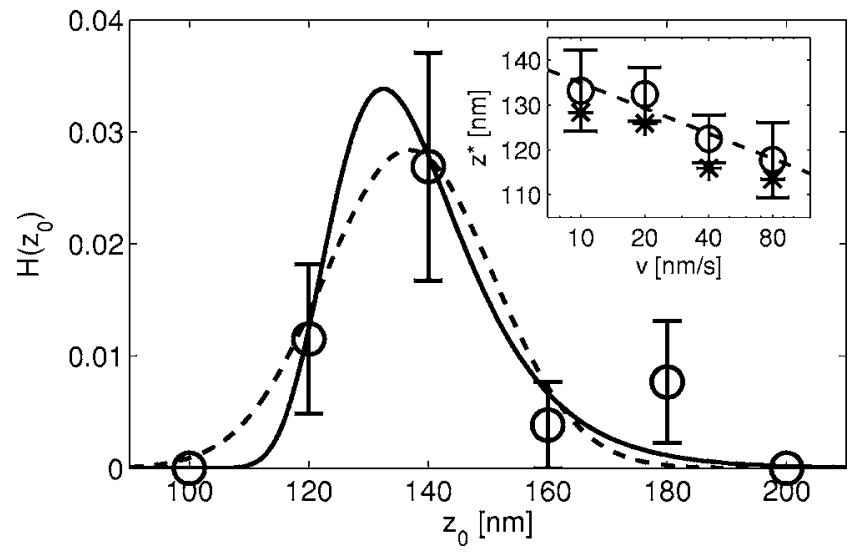

FIG. 4. Histogram (bin width $=20 \mathrm{~nm}$ ) of jumps at approach speed $20 \mathrm{~nm} / \mathrm{s}$. The solid line shows a fit of Eq. (22). For comparison, a Gaussian fit (dashed line) is also shown. Inset: $z^{*}$ as a function of approach velocity. Circles (with error bars) denote experimentally obtained data. Asterisks indicate Langevin simulation data. The dashed line is a linear fit to the experimental data. 
locities shown here. Using Eq. (21) we find $\alpha=0.12 \mathrm{~nm}^{-1}$ from the slope of the inset in Fig. 4. Fitting experimental data we generally observe that the values of $\alpha$ found using Eq. (22) are lower than when Eq. (21) is used. This is due to experimental uncertainties which tend to broaden the observed jump distributions.

If the total energy is as described by Eq. (1), the only free parameter in our model is the Hamaker constant $A$. In order to determine this parameter experimentally, we solve Eq. (20) where both $\Delta V$ and $f_{0}$ implicitly depend on $A$. Using the results from the experiments depicted in Fig. 4, $\alpha=0.12 \mathrm{~nm}^{-1}$, and the typical velocity, $\bar{v}=28 \mathrm{~nm} / \mathrm{s}$, Eq. (20) was solved numerically, resulting in a value of $A=1.7 \times 10^{-20} \mathrm{~J}$ which can be compared to $A=1.5 \times 10^{-20} \mathrm{~J}$ found in [8]. To further compare experiments to simulations, the experimentally obtained values of $A$ and $\kappa$ were inserted in a Langevin simulation. The result of the simulation is shown by asterisks in the inset of Fig. 4. There is good agreement between experiments and Langevin simulations as the typical jump lengths from the simulations were only $6 \mathrm{~nm}$ less than experimentally observed.

\section{EXTENSIONS AND ERROR CONSIDERATIONS}

Retardation effects of the van der Waals attraction have been neglected in Eq. (1). A more detailed analysis would allow the Hamaker constant to be distance dependent. However, our comparison with Langevin simulations shows that the simple model explains our data sufficiently well. The Hamaker constant we find should be interpreted as an average value in the observed interval.

The simplest interpretation of a jump experiment would be to neglect the thermal energy of the probe. However, this induces a systematic error in the determination of the Hamaker constant. Here, we investigate the size of this systematic error: If one neglects the influence of the thermal energy, the colloidal particle would escape when the energy barrier is zero. This corresponds to applying Eq. (6) and (7) to determine the Hamaker constant $A$. When the temperature vanishes, $T=0$, the observed jump length must be equal to $z_{c}$. The mean jump length in the experiment is found from Fig. 1(b) to be around $135 \mathrm{~nm}$. Solving Eq. (7) for $A$ we get:

$$
A=\frac{8 \kappa z_{c}^{3}}{9 R} \simeq 4 \times 10^{-20} \mathrm{~J} .
$$

As the correct answer is $A=1.5 \times 10^{-20} \mathrm{~J}$, this result represents an overestimation of the strength of the interaction potential by a factor of almost three.

The error is even larger if the experimentally observed jump length is interpreted as being equal to $\zeta$. If this assump- tion is applied to the analyses of our experimental data, the value of the Hamaker constant would be overestimated by nearly one order of magnitude: Since $z_{c}=(3 / 2) \zeta \simeq 200 \mathrm{~nm}$ we would get $A \simeq 13 \times 10^{-20} \mathrm{~J}$.

In any case, the dependence of $A$ on $z_{c}^{3}$ induces a large uncertainty in estimates based on the critical apparatus position.

In similar studies using atomic force microscopy (AFM) [13], the thermal energy of the probe is typically neglected. The stiffness of an AFM cantilever is $\kappa \simeq 10-100 \mathrm{pN} / \mathrm{nm}$, and even for a high approach speed, $v=1000 \mathrm{~nm} / \mathrm{s}$, the systematic overestimation of the interaction energy amounts to a factor of 1.5 on $A$.

The framework presented in this paper may be generalized to study a variety of nanoscale systems. Here, we demonstrated it for a colloidal system and our experimental procedure is directly applicable to quantitative measurements of biological phenomena such as cell adhesion. Also topics in condensed matter physics such as, e.g., the stability of liquid interfaces may benefit from a similar analysis: In the work described in Refs. [14,15] it is likely that the velocity of retraction influences the observed critical distances.

\section{CONCLUSION}

A quantitative description of small-scale systems out of thermal equilibrium poses a challenge to the physical community. We attack this problem and devise a way to quantitatively deal with the escape of a particle in a changing energy landscape. Experimentally, this is tested by having a colloid in an optical trap approaching an attractive surface to which the colloid eventually jumps. The experiment could equally well have been an AFM cantilever approaching a surface. Theoretically, we treat this scenario by a model based on Kramers equation. The validity of this approach is tested by Langevin simulations as well as by comparison to experiments. In particular, we show how to find the constants which describe the underlying interaction potential. In our particular test case, the Hamaker constant would have been overestimated by a factor of roughly 3 , if thermal fluctuations had not been taken into account.

\section{ACKNOWLEDGMENTS}

We would like to thank Jesper Ferkinghoff-Borg, Per Lyngs Hansen, Poul Martin Hansen, Esben Thormann and Håkan Wennerström for fruitful discussions. Also, we acknowledge financial support from the Danish Research Councils, the NKT Research Academy, the Carlsberg Foundation, and BioNET by the Villum Kahn Rasmussen Foundation.
[1] C. Jarzynski, Phys. Rev. Lett. 78, 2690 (1997).

[2] G. Crooks, Phys. Rev. E 60, 2721 (1999).

[3] J. Liphardt, S. Dumont, S. Smith, I. Tinoco, and C. Bustamante, Science 296, 1832 (2002).
[4] C. Collin, R. Ritort, C. Jarzynski, S. Smith, I. Tinoco, and C. Bustamante, Nature (London) 437, 231 (2005).

[5] H. Kramers, Physica (Amsterdam) 7, 284 (1940).

[6] L. Oddershede, S. Grego, S. Nørrelykke, and K. Berg- 
Sørensen, Probe Microsc. 2, 129 (2001).

[7] J. Dreyer, K. Berg-Sørensen, and L. Oddershede, Appl. Opt. 43, 1991 (2004).

[8] P. M. Hansen, J. K. Dreyer, J. Ferkinghoff-Borg, and L. Oddershede, J. Colloid Interface Sci. 287, 561 (2005).

[9] Kirstine Berg-Sørensen and Henrik Flyvbjerg, Rev. Sci. Instrum. 75, 594 (2004).

[10] J. N. Israelachvili, Intermolecular and Surface Forces, 2nd ed. (Academic Press, London, 1992).
[11] H. Brenner, Chem. Eng. Sci. 16, 242 (1961).

[12] J.-P. Bouchaud and M. Mézard, J. Phys. A 30, 7997 (1997).

[13] B. Gady, D. Schleef, R. Reifenberger, and D. Rimay, J. Adhes. 67, 291 (1998).

[14] P. Dagastine and L. White, J. Colloid Interface Sci. 247, 310 (2002).

[15] N. Maeda, J. N. Israelachvili, and M. M. Kohonen, Proc. Natl. Acad. Sci. U.S.A. 100, 803 (2003). 